\title{
BMJ Open Short-term outcomes of rapid initiation of antiretroviral therapy among HIV- positive patients: real-world experience from a single-centre retrospective cohort in Taiwan
}

\author{
Yi-Chia Huang, ${ }^{\oplus 1}$ Hsin-Yun Sun, ${ }^{2}$ Yu-Chung Chuang, ${ }^{2}$ Yu-Shan Huang, ${ }^{2}$ \\ Kuan-Yin Lin, ${ }^{3}$ Sung-Hsi Huang, ${ }^{1,4}$ Guan-Jhou Chen, ${ }^{5}$ Yu-Zheng Luo, ${ }^{6}$ \\ Pei-Ying Wu, ${ }^{6}$ Wen-Chun Liu, ${ }^{2}$ Chien-Ching Hung, ${ }^{2,4,7,8}$ Shan-Chwen Chang ${ }^{2}$
}

To cite: Huang $\mathrm{Y}-\mathrm{C}$, Sun $\mathrm{H}-\mathrm{Y}$, Chuang Y-C, et al. Short-term outcomes of rapid initiation of antiretroviral therapy among HIV-positive patients: real-world experience from a single-centre retrospective cohort in Taiwan. BMJ Open 2019;9:e033246. doi:10.1136/ bmjopen-2019-033246

- Prepublication history and additional material for this paper are available online. To view please visit the journal (http:// dx.doi.org/10.1136/bmjopen2019-033246).

Received 30 July 2019 Revised 05 September 2019 Accepted 06 September 2019

Check for updates

(C) Author(s) (or their employer(s)) 2019. Re-use permitted under CC BY-NC. No commercial re-use. See rights and permissions. Published by BMJ.

For numbered affiliations see end of article.

Correspondence to Dr Chien-Ching Hung; hcc0401@ntu.edu.tw

\section{ABSTRACT}

Objectives Rapid initiation of antiretroviral therapy (ART) engenders faster viral suppression but with suboptimal rates of durable viral suppression and engagement in care, as reported by clinical trials in resource-limited settings. Real-world experience with rapid ART initiation remains limited in resource-rich settings.

Design Retrospective cohort study.

Setting A tertiary hospital in metropolitan Taipei, Taiwan. Participants We included 631 patients newly diagnosed as having HIV infection between March 2014 and July 2018.

Main outcome measures Rapid ART initiation was defined as starting ART within 7 days after HIV diagnosis confirmation. HIV diagnosis, ART initiation and viral suppression dates and clinical outcome data were collected by reviewing medical records. The rates of loss to follow-up (LTFU), engagement in care and virological rebound at 12 months were compared between patients with rapid ART initiation and those with standard initiation.

Results Rapid ART initiation increased from $33.8 \%$ in 2014 to $68.3 \%$ in 2017, and the median interval between HIV diagnosis and viral suppression (HIV RNA load $<200$ copies $/ \mathrm{mL}$ ) decreased from 138 to 47 days. Patients with rapid ART initiation had a significantly higher rate of engagement in care at 12 months than did those with standard initiation ( $88.3 \%$ vs $79.0 \% ; \mathrm{p}=0.002$ ). Patients aged $<30$ years had a higher risk of LTFU (HR: 2.19; 95\% Cl 1.20 to 3.98); and rapid ART initiation was associated with a lower risk of LTFU (HR: $0.41 ; 95 \% \mathrm{Cl} 0.24$ to 0.83 ). Patients aged $<30$ years were more likely to acquire incident sexually transmitted infections (STIS) before achieving viral suppression.

Conclusions Rapid ART initiation was associated with a higher rate of engagement in care at 12 months and shortened interval from diagnosis to HIV suppression. Delayed ART initiation may increase onwards HIV transmission considering the high rates of STIs. Ethics approval The study was approved by the Research Ethics Committee of National Taiwan University Hospital (Registration No. 201003112R).
Strengths and limitations of this study

- This study provides real-world data on the shortterm outcomes of rapid initiation of antiretroviral therapy (ART) under a national policy at a single centre in a developed country.

- This study examined and compared both virological and clinical outcomes at 12 months after rapid versus standard ART initiation.

- This is the first study to compare the rates of incident sexually transmitted infections before achievement of viral suppression between two ART strategies.

- The present study is limited by its retrospective study design and could not determine the shared decision-making process between treating physicians and patients regarding the time to initiate ART.

- The study does not provide long-term outcomes beyond 48 weeks of ART initiation.

\section{INTRODUCTION}

The TEMPRANO and START studies have provided evidence that early initiation of combination antiretroviral therapy (cART) is associated with a more favourable prognosis; subsequently, the WHO recommended in 2015 that all patients be treated with cART once HIV infection is diagnosed. ${ }^{1-5}$ At the population level, starting antiretroviral therapy (ART) soon after HIV diagnosis can prevent onwards HIV transmission ('treatment as prevention'), ${ }^{6}$ especially when high rates of incident sexually transmitted infections (STIs) are observed among patients diagnosed as having acute HIV infection, which are considered biomarkers of onwards transmission. ${ }^{7}$ In the PARTNER 1 and PARTNER 2 studies, no within-couple HIV transmission occurred through condomless sex between serodiscordant heterosexuals or 


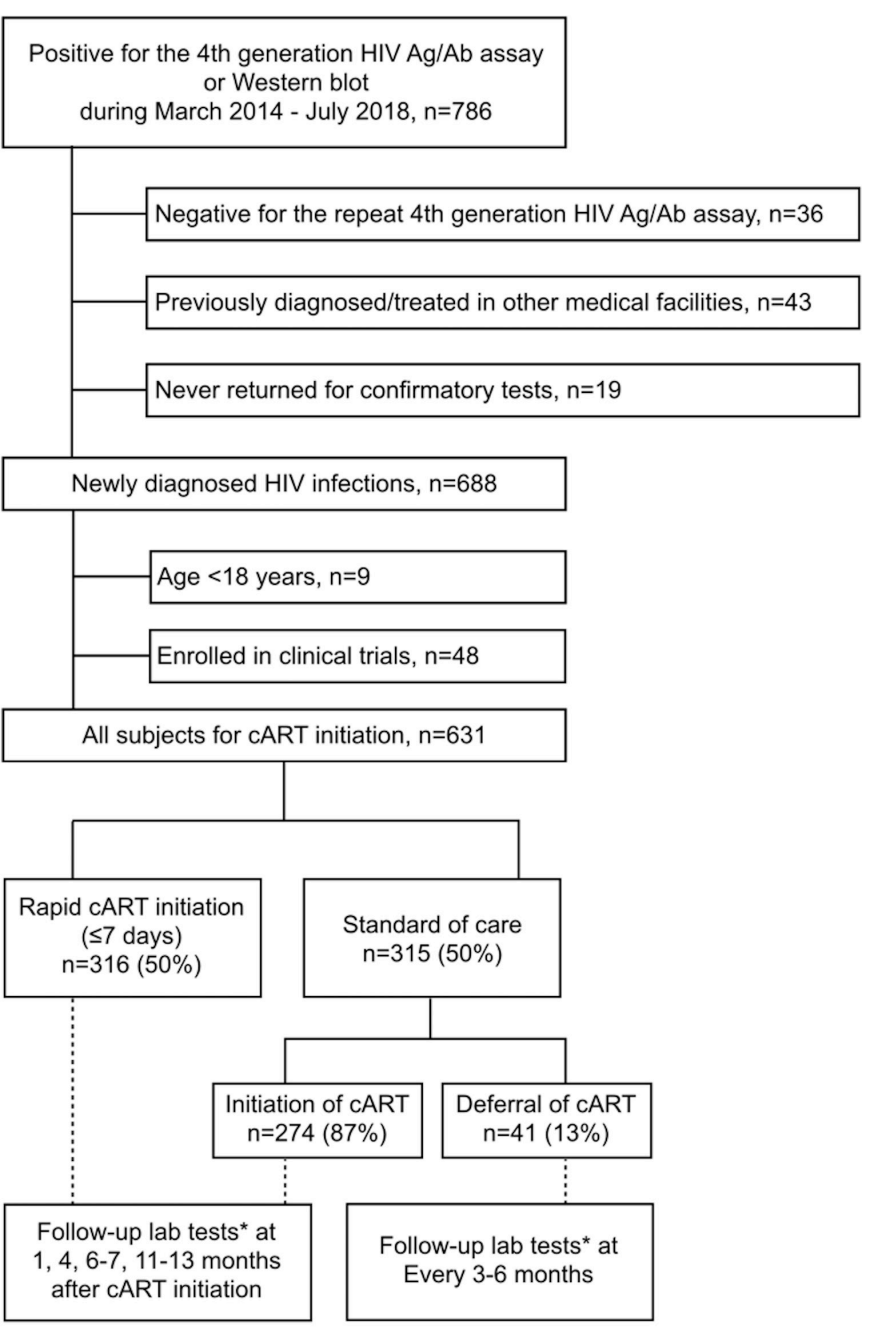

*HIV viral load, CD4, CD8, RPR

Figure 1 Study flow. Ab, antibody; Ag, antigen; cART, combination antiretroviral therapy; RPR, rapid plasma reagin.

between male homosexual couples when the HIV-positive partners were on suppressive ART. ${ }^{8}$ However, although the WHO has recommended a 'treat-all' policy since $2015,{ }^{1} 1.8$ million people were still newly infected with HIV in 2017. ${ }^{10}$ The substantial loss of patients during linkage to medical care, ART initiation and engagement in care among the most vulnerable populations has been a major concern in ART scale-up efforts. ${ }^{11}{ }^{12}$ Therefore, the concept of rapid ART initiation-defined as starting ART within 7 days or even on the same day after HIV diagnosis- has been introduced to improve the HIV care continuum. ${ }^{13}$

In several proof-of-concept clinical trials conducted in developing countries to investigate the feasibility and efficacy of rapid ART initiation, ${ }^{14-18}$ patients who received rapid ART initiation had higher rates of ART uptake and faster viral suppression than did those for whom ART was initiated according to standards of care. For example, a trial of single-visit ART initiation in South Africa resulted in a higher rate of viral suppression at 10 months in participants assigned to rapid ART initiation compared with other participants $(64 \%$ vs $51 \%) .{ }^{15}$ On the basis of these clinical trials, the WHO issued guidelines on rapid ART initiation in $2017 .{ }^{19}$ However, $29 \%-50 \%$ of patients enrolled in these studies did not achieve viral suppression 10-12 months after rapid ART initiation. ${ }^{14-16} 18$ Moreover, despite the clinical trial settings, the rates of engagement in care were low, ranging from $63 \%$ to $81 \%$ in the rapid ART initiation arm and from $48 \%$ to $71 \%$ in the standard initiation arm. ${ }^{14-16} 18$

In contrast to the findings of the aforementioned trials, studies conducted in resource-rich settings have shown a consistently shorter time to viral suppression and higher rates of engagement in care, although the numbers of participants are considerably smaller. ${ }^{132021}$ The San Francisco RAPID Study found that the median time to viral suppression was only 65 days among 39 patients who adopted same-day ART initiation. ${ }^{13}$ From the Acute HIV Cohort in the UK, the median time to viral suppression was only 41 days among 36 patients who started integrase strand transfer inhibitor (InSTI)-based regimens on the same day after HIV diagnosis; moreover, nearly all patients (99\%) achieved viral suppression and were engaged in care at week $24 .{ }^{21}$ Data on rapid ART initiation in developed countries are based on small cohort studies through project-based schemes. Thus, the results may not be generalisable to other real-world settings.

The aim of the present study was to evaluate the outcomes of rapid ART initiation delivered by public sectors in a developed country.

\section{METHODS \\ Study population and setting}

In this retrospective cohort study, we included all patients who tested positive for HIV through the fourth-generation HIV antigen/antibody combo assay followed by confirmation using Western blotting or a nucleic-acid amplification test (NAAT) at National Taiwan University hospital in Taiwan between 1 March 2014 and 31 July 2018. Individuals who tested negative for HIV, received HIV diagnosis at other healthcare facilities, or never returned for confirmatory tests were excluded. Patients aged younger than 18 years or patients participating in clinical trials were also excluded (figure 1). Patients were divided into four groups according to the date of HIV diagnosis: group 1, March 2014 to March 2015 (calendar year, 2014); group 2, April 2015 to March 2016 (2015); group 3, April 2016 to March 2017 (2016); and group 4, April 2017 to July 2018 (2017). Medical records were reviewed to collect information on clinical characteristics and laboratory test results. The need for informed consent was waived because of the retrospective design of the study.

In 2013, the Taiwan Centers for Disease Control increased the treatment threshold of $\mathrm{CD} 4$ count for ART initiation from 350 to 500 cells $/ \mu \mathrm{L}$. In 2016, Taiwan adopted the treat-all policy recommended by the WHO, and in January 2018, rapid ART initiation was implemented. In 2016, Taiwan's national HIV treatment 
guidelines recommended the following three single-tablet regimens for treatment: coformulated tenofovir disoproxil fumarate/emtricitabine (FTC)/efavirenz, tenofovir disoproxil fumarate /FTC/rilpivirine and abacavir/ lamivudine/dolutegravir. The coformulated elvitegravir/ cobicistat/FTC/tenofovir alafenamide regimen was not available at our hospital until March 2018.

\section{HIV diagnostic algorithm and standardised laboratory assessment in Taiwan}

Before 2018, confirmation of HIV diagnosis required reactive immunoassays using the HIV antigen/antibody combo assay (ARCHITECT; Abbott Diagnostics, Weisbaden, Germany), followed by Western blot assays for HIV-1 or HIV-2 (New LAV Blot I or II kits; Bio-Rad, Les Ulis, France). The NAAT for confirmation of HIV infection was reserved for patients with indeterminate Western blot results, pregnant women or individuals presenting with acute HIV infection who tested negative in the combo assay. ${ }^{22}$ Since 2018, the NAAT has been included as a confirmatory test for HIV infection. In the present study, the date of HIV diagnosis was defined as the earliest date of positive NAAT or Western blot results. ${ }^{23}$

According to the national HIV treatment guidelines, patients on cART require CD4 count and plasma HIV RNA load (PVL) monitoring 1 month after ART initiation and then every 3 months within the first year. For patients who defer ART, monitoring of CD4 count and PVL every 3-6 months is recommended. The guidelines suggest that testing of the rapid plasma reagin titer be performed annually or on an as-needed basis when patients present with symptoms suggestive of syphilis or other STIs (figure 1).

\section{Outcomes}

In this study, the interval between HIV diagnosis and ART initiation was calculated from the date of HIV diagnosis to the date of cART initiation. The time to viral suppression was defined as the interval between the date of HIV diagnosis and the date of virally suppressed plasma sample acquisition. Outcome was determined at 12 months after the patient's engagement in care. Viral suppression was defined as a PVL of $<200$ copies/mL after ART initiation. Loss to follow-up (LTFU) was defined when a patient had not attended any clinic visit for 180 days at the time of outcome assessment (12 months).$^{24}$ Virological rebound was defined as a confirmed PVL of $\geq 200$ copies $/ \mathrm{mL}$ after the achievement of viral suppression. Incident STIs diagnosed before the achievement of viral suppression were recorded and considered a marker of potentially onwards transmission of HIV. Syphilis was defined when a fourfold elevation of rapid plasma reagin titer or consistent clinical symptoms were detected. To detect other STIs, cultures for Neisseria gonorrhoeae, an antigen test for Chlamydia trachomatis, and a serum indirect hemagglutination test or direct microscopy of stool samples for Entamoeba histolytica were performed as clinically indicated. The diagnosis of genital warts was made by inspection. The incidence of any STI was defined as the total event of incident STIs occurring before viral suppression was achieved divided by the total person-years of viremia in this cohort.

\section{Statistical analysis}

All statistical analyses were performed using STATA software V.14.0s/E. Variables with a normal distribution are presented as mean $\pm \mathrm{SD}$, and variables with a skewed distribution are presented as median and IQR. For baseline CD4, PVL, syphilis, hepatitis B virus and hepatitis C virus, missing variables were excluded from the analysis. Box plots were used to describe data with outliers. Categorical variables were compared using the $\chi^{2}$ test and Fisher's exact test. Continuous variables were compared using one-way analysis of variance or the Kruskal-Wallis test, depending on the distribution. All tests were two-tailed, and a $p$ value of $<0.05$ was considered statistically significant. The last-observation-carried-forward imputation was performed when PVL data were missing at week 6 , month 3 , month 6 and month 12. Cox-regression analysis and Kaplan-Meier analysis were used to evaluate factors associated with LTFU, virological rebound and incident STIs before viral suppression.

\section{Patient and public involvement}

Due to the retrospective study design, patients were not involved in the process of research question development, recruitment, study conduction or outcome measurement. The finding of this study will be reported to patients and the public through academic conferences and an open-access journal.

\section{RESULTS}

\section{Patient characteristics}

During the 4-year study period, 688 patients were newly diagnosed as having HIV infection. Of these patients, we included 631 for analysis (figure 1), and we excluded patients who tested negative in a repeated immunoassay $(\mathrm{n}=36)$, received HIV diagnosis at other healthcare facilities $(n=43)$, never returned for confirmatory tests $(n=19)$, were aged younger than 18 years $(n=9)$ or participated in clinical trials $(n=48$; figure 1$)$. According to the date of HIV diagnosis, 201, 190, 117 and 123 patients were included in groups 1 (calendar year 2014), 2 (2015), 3 (2016) and 4 (2017), respectively (table 1 ). The majority of the included patients were men who have sex with men (96.2\%; mean age $=32.8$ years). The proportions of late presenters $(\mathrm{CD} 4<200$ cells $/ \mu \mathrm{L})$ were consistently higher than $25 \%$ in the four groups. The median CD4 count was lower in group 3, whereas the proportion of acute HIV infection was higher in group $2(\mathrm{p}=0.022)$. The percentages of coinfections with viral hepatitis or syphilis before ART initiation did not differ significantly among the four groups of patients. 
Table 1 Baseline characteristics of patients newly diagnosed as having HIV infection in the four calendar groups

\begin{tabular}{|c|c|c|c|c|c|c|}
\hline & $\begin{array}{l}\text { All } \\
(n=631)\end{array}$ & $\begin{array}{l}\text { Group } 1 \\
(2014 / 03- \\
2015 / 03) \\
(n=201)\end{array}$ & $\begin{array}{l}\text { Group } 2 \\
(2015 / 04- \\
2016 / 03) \\
(n=190)\end{array}$ & $\begin{array}{l}\text { Group } 3 \\
(2016 / 04- \\
2017 / 03) \\
(n=117)\end{array}$ & $\begin{array}{l}\text { Group } 4 \\
(2017 / 04- \\
2018 / 07) \\
(n=123)\end{array}$ & $P$ value \\
\hline Age, mean (SD), years & $32.8(9.5)$ & $32.4(9.9)$ & $33.5(9.7)$ & $31.6(9.4)$ & 33.7 (8.8) & 0.223 \\
\hline Male, $\mathrm{n}(\%)$ & $622(98.6)$ & $197(98.0)$ & $188(98.9)$ & $116(99.1)$ & $121(98.4)$ & 0.691 \\
\hline $\begin{array}{l}\text { Men who have sex with men, } \\
\mathrm{n}(\%)\end{array}$ & 607 (96.2) & $192(95.5)$ & $182(95.8)$ & $114(97.4)$ & $119(96.7)$ & 0.777 \\
\hline $\begin{array}{l}\text { PVL at baseline, median } \\
\text { (IQR), } \log _{10} \text { copies } / \mathrm{ml}\end{array}$ & $5.0(4.5-5.5)$ & $5.0(4.5-5.5)$ & $5.0(4.5-5.4)$ & $5.2(4.6-5.8)$ & $4.9(4.5-5.5)$ & 0.017 \\
\hline $\begin{array}{l}\text { CD4 count at baseline, } \\
\text { median (IQR), cells/ } \mu \mathrm{L}\end{array}$ & 295 (133-442) & $323(133-483)$ & $286(135-452)$ & $225(79-362)$ & $318(160-438)$ & 0.009 \\
\hline 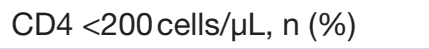 & $209(34.4)$ & $56(27.9)$ & $67(35.3)$ & $48(41.0)$ & $38(32.2)$ & 0.105 \\
\hline Acute HIV infection, $\mathrm{n}(\%)$ & 77 (12.2) & $19(9.5)$ & $34(17.9)$ & $15(12.8)$ & $9(7.32)$ & 0.022 \\
\hline Anti-HCV positivity, n (\%) & $29(4.8)$ & $7(3.5)$ & $7(3.7)$ & $8(6.8)$ & $7(5.7)$ & 0.484 \\
\hline HBsAg positivity, n (\%) & $52(8.7)$ & $14(7.0)$ & $19(10.1)$ & $10(8.5)$ & $9(7.3)$ & 0.767 \\
\hline Syphilis, n (\%) & $120(20.2)$ & $29(15.8)$ & 37 (20.6) & 23 (20.5) & $31(26.1)$ & 0.185 \\
\hline
\end{tabular}

HBsAg, hepatitis B virus surface antigen; HCV, hepatitis C virus; PVL, plasma HIV RNA load.

\section{HIV care continuum and virological response to art}

Overall, 316 patients $(50.1 \%)$ initiated cART within 7 days of HIV diagnosis (figure 1). The proportion of acute HIV infection $(\mathrm{p}=0.022)$ and late presenters $(\mathrm{p}<0.001)$ were significantly higher among patients with rapid ART initiation than among those than without rapid ART initiation (online supplementary table 1 ). In each group, $>92 \%$ of patients started ART during the study period (table 2). The proportions of rapid ART initiation increased significantly from $33.8 \%$ in group 1 (calendar year 2014) to $68.3 \%$ in group 4 (calendar year 2017; $\mathrm{p}<0.001$ ). Initiation of InSTI-based regimens also increased significantly from $5.9 \%$ in group $1 \%$ to $69.8 \%$ in group 4 (table 2). The median (IQR) interval from confirmed HIV diagnosis to ART initiation decreased significantly from 21 (12-105) days in group 1 to 7 (5-11) days in group 4 $(\mathrm{p}<0.001$; figure 2$)$. Of the patients who initiated cART, only $25.1 \%$ in group 1 achieved viral suppression $(<200$ copies $/ \mathrm{mL}$ ) within 6 weeks of treatment, whereas $52.9 \%$ in group 4 achieved viral suppression within 6 weeks of treatment $(\mathrm{p}<0.001$; online supplementary figure 1$)$. The proportion of patients who achieved viral suppression at 3-4 months of cART increased from $41.7 \%$ in group $1 \%$ to $61.3 \%$ in group $4(p=0.001)$, whereas the proportions of viral suppression at 6-7 months of cART were similar among the four groups $(\mathrm{p}=0.868$; online supplementary figure 1). The median (IQR) interval from HIV diagnosis to viral suppression also shortened from 138 (104-249) days in group 1 to 47 (36-111) days in group 4 ( $\mathrm{p}<0.001$; figure 3).

\section{Outcomes and associated factors}

Compared with patients without rapid ART initiation, those with rapid ART initiation had a higher rate of engagement in care $(88.3 \%$ vs $79.0 \% ; \mathrm{p}=0.002)$ and a lower rate of LTFU ( $4.7 \%$ vs $10.5 \%$; $\mathrm{p}=0.007$ ) at 12 months after HIV diagnosis (table 3). The rates of virological rebound, death and transfer out between

Table 2 ART initiation among the four calendar groups

\begin{tabular}{lccccc}
\hline & $\begin{array}{l}\mathbf{2 0 1 4 / 0 3 -} \\
\mathbf{2 0 1 5 / 0 3} \\
(\mathbf{n = 2 0 1 )}\end{array}$ & $\begin{array}{l}\mathbf{2 0 1 5 / 0 4 -} \\
\mathbf{2 0 1 6 / 0 3 2 3} \\
(\mathbf{n = 1 9 0 )}\end{array}$ & $\begin{array}{l}\mathbf{2 0 1 6 / 0 4 -} \\
\mathbf{2 0 1 7 / 0 3} \\
(\mathbf{n = 1 1 7})\end{array}$ & $\begin{array}{l}\mathbf{2 0 1 7 / 0 4 -} \\
\mathbf{2 0 1 8 / 0 7} \\
(\mathbf{n = 1 2 3})\end{array}$ & P value \\
\hline ART initiation, n (\%) & $187(93.0)$ & $177(93.2)$ & $108(92.3)$ & $119(96.7)$ & 0.283 \\
Rapid ART initiation, n (\%) & $68(33.8)$ & $101(53.2)$ & $63(53.8)$ & $84(68.3)$ & $<0.001$ \\
Third agent of ART used & & & & & \\
nNRTI, n (\%) & $171(91.4)$ & $166(93.8)$ & $85(78.7)$ & $36(30.3)$ & $<0.001$ \\
PI, n (\%) & $4(2.1)$ & $2(1.1)$ & 0 & 0 & 0.265 \\
InSTI, n (\%) & $11(5.9)$ & $9(5.1)$ & $23(21.3)$ & $83(69.8)$ & $<0.001$ \\
\hline
\end{tabular}

ART, antiretroviral therapy; nNRTI, non-nucleoside reverse-transcriptase inhibitors; PI, protease inhibitor; InSTI, integrase strand transfer inhibitor 


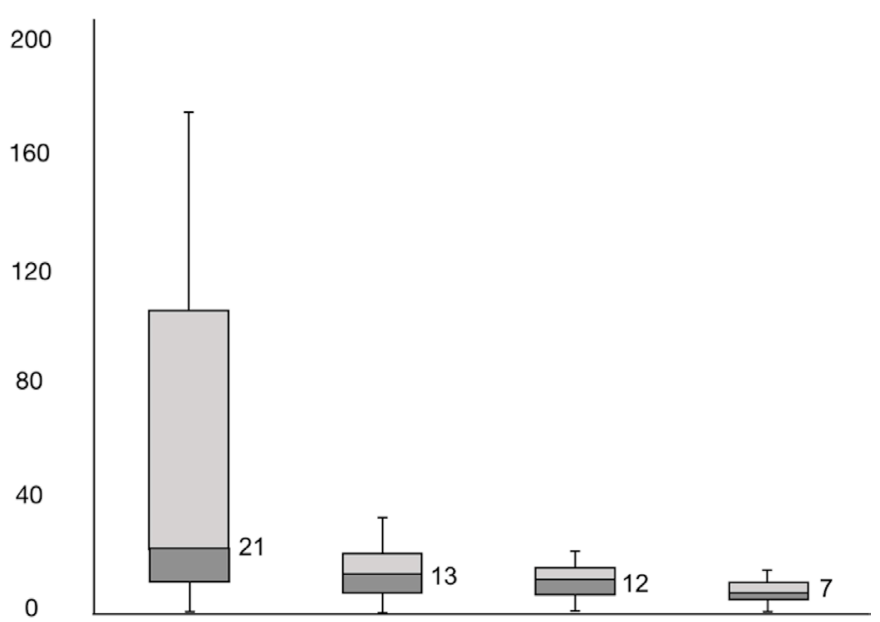

2014/03-2015/03 2015/04-2016/03 2016/04-2017/03 2017/04-2018/07

Figure 2 Intervals (in days) between confirmed HIV diagnosis and ART initiation in the four groups. ART, antiretroviral therapy.

patients with and without rapid ART initiation did not differ significantly (table 3 ). In our Cox regression analysis (table 4), patients aged younger than 30 years were more likely to have LTFU (HR: 2.19; $95 \%$ CI 1.20 to 3.98 ; $\mathrm{p}=0.01$ ), whereas patients with rapid ART initiation were less likely to have LTFU (HR: $0.41 ; 95 \%$ CI 0.24 to 0.83 ; $\mathrm{p}=0.01$ ). The calendar year of HIV diagnosis, risk group of HIV transmission, baseline CD 4 count $<200$ cells $/ \mu \mathrm{L}$ and presentation of acute HIV infection were not significantly associated with LTFU (table 4). The Kaplan-Meier plots of LTFU stratified by age $<30$ years and timing of ART initiation are shown in figure 4 . We did not observe any factor associated with virological rebound among patients engaging in care (online supplementary tables 2 and 3$)$.

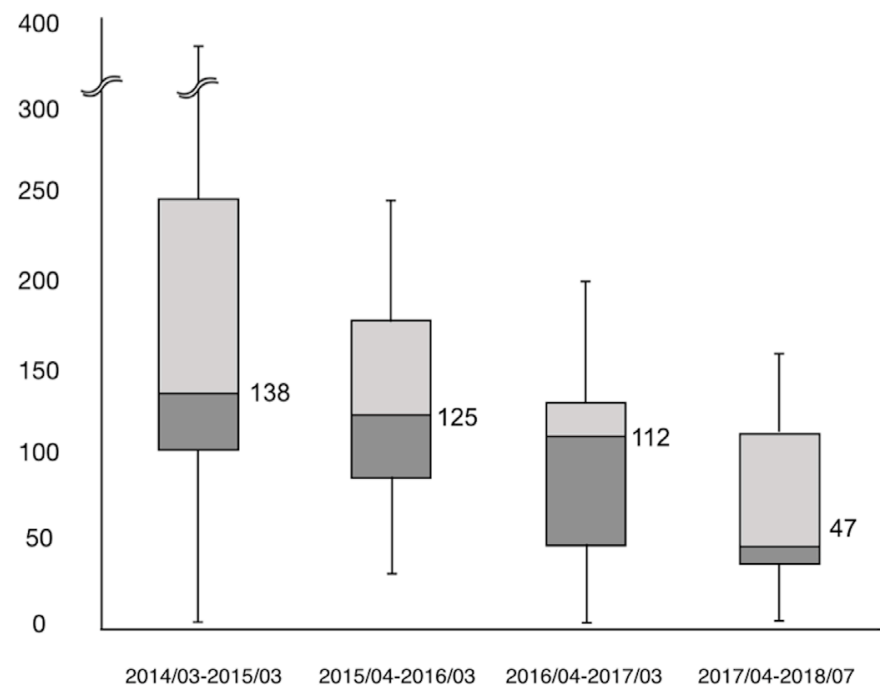

Figure 3 Intervals (in days) between confirmed HIV diagnosis and PVL $<200$ copies/mL in the four groups. PVL, plasma HIV RNA load.
Table 3 Outcome at 12 months in patients with rapid ART and those with standard initiation

\begin{tabular}{|c|c|c|c|}
\hline & $\begin{array}{l}\text { Rapid ART } \\
\text { initiation } \\
(n=316)\end{array}$ & $\begin{array}{l}\text { Standard of } \\
\text { care } \\
(n=315)\end{array}$ & $P$ value \\
\hline Death, n (\%) & $5(1.6)$ & $9(2.9)$ & 0.296 \\
\hline $\begin{array}{l}\text { Engagement in care, } \\
\mathrm{n}(\%)\end{array}$ & 279 (88.3) & $249(79.0)$ & 0.002 \\
\hline $\begin{array}{l}\text { Virological rebound, } \\
\mathrm{n}(\%)\end{array}$ & $8(2.5)$ & $14(4.4)$ & 0.202 \\
\hline $\begin{array}{l}\text { Loss to follow-up, } \\
n(\%)\end{array}$ & $15(4.7)$ & $33(10.5)$ & 0.007 \\
\hline Transfer out, n (\%) & $9(2.8)$ & $10(3.2)$ & 0.821 \\
\hline
\end{tabular}

*Definition of virological rebound: a confirmed PVL $\geq 200$ copies $/ \mathrm{mL}$ after achieving viral suppression.

ART, antiretroviral therapy.

\section{Incidence of STIs before viral suppression}

After a median observation period of 0.29 years (IQR: $0.10-0.33$ ) between diagnosis and viral suppression, eight cases of syphilis and eight other symptomatic STIs were identified among patients with rapid ART initiation, resulting in an incidence of 18.8 per 100 personyears of follow-up (PYFU; online supplementary table 4). For patients without rapid ART initiation, we observed 37 cases of syphilis and 15 other symptomatic STIs after a median observation of 0.36 years (IQR: 0.13-0.67), resulting in an incidence of 28.3 per 100 PYFU (adjusted HR (aHR): 1.08 ; $95 \%$ CI 0.59 to 2.00 ). In the Cox proportional hazards model (online supplementary table 5), we examined the association between incident STIs and rapid ART initiation, age, any STIs at baseline, CD4 count and PVL at HIV diagnosis. We found that the timing of ART initiation was not associated with incident STIs $(\mathrm{p}=0.802$; online supplementary table 5 and online supplementary figure 2), and the only factor associated with incident STIs was age $<30$ years (aHR: 2.78 ; 95\% CI 1.53 to 5.06 ; $\mathrm{p}=0.001$; online supplementary table 5 and figure 3 ).

\section{DISCUSSION}

We conducted this retrospective cohort study on cART initiation among patients newly diagnosed as being HIV positive in a real-world setting. We found an increasing trend of patients initiating ART within 7 days of confirmed HIV diagnosis during the 4-year study period. Rapid ART initiation was associated with a shorter time to suppression of HIV replication, particularly among patients who initiated InSTI-based cART; such rapid initiation was also associated with a higher rate of engagement in care along with a lower rate of LTFU at 12 months. In addition, higher rates of incident STIs and LTFU were observed among patients aged younger than 30 years.

In randomised trials conducted in resource-limited settings, ${ }^{151618}$ the rates of LTFU were high (20\%-30\%) in both same-day and rapid ART initiation arms despite 
Table 4 Cox regression model of HR for loss to follow-up

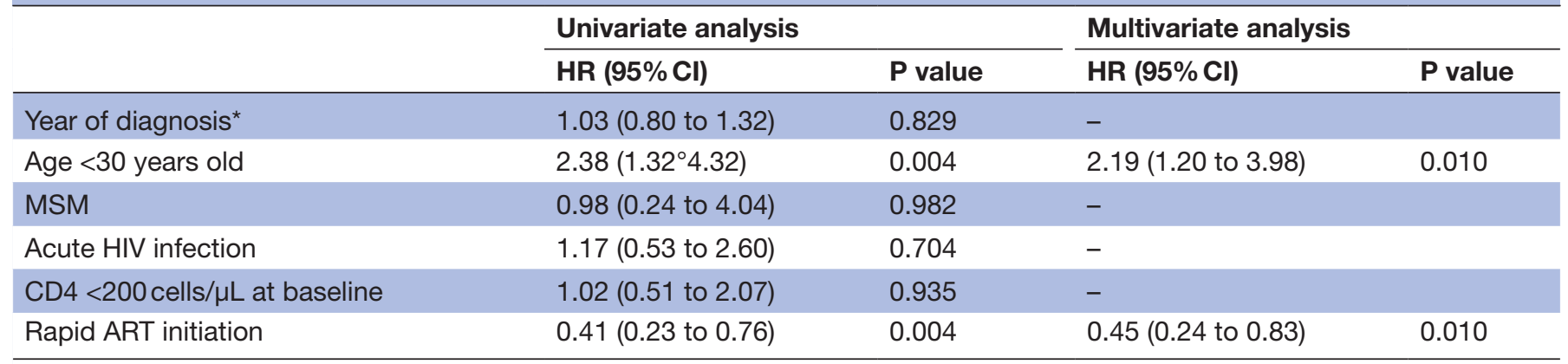

*Comparisons made between the four groups of patients diagnosed during March 2014 to March 2015, April 2015 to March 2016 , April 2016 to March 2017 and April 2017 to July 2018.

MSM, men who have sex with men; ART, antiretroviral therapy.

the clinical trial settings; these high rates can primarily be attributed to frequent clinic transferal, refusal to attend clinics, lack of time to attend clinics due to lack of transportation, financial pressure and forced displacement. ${ }^{25}$ Furthermore, a meta-analysis of observational studies revealed an increasing trend of LTFU at 6 months among patients who initiated ART within 7 days of HIV diagnosis (response rate: $1.85 ; 95 \%$ CI 0.96 to 3.55 ). ${ }^{26}$ By contrast, studies conducted in recourse-rich settings have reported low and similar rates of LTFU between patients who adopted rapid ART initiation $(<10 \%)$ and those who initiated ART according to standards of care $(<15 \%){ }^{13}{ }^{21}$ Our retrospective cohort study demonstrated that patients adopting rapid ART initiation were more likely to continue engagement in care and to have a significantly lower rate of LTFU compared with those without rapid ART initiation. The discrepancies in the findings reported by our study and the aforementioned studies could be attributed to the different study settings and designs, patient populations, HIV infection status, cART accessibility, patients' knowledge and peer experience with ART. ${ }^{27} 28$ However, the retrospective design of our study precluded us from identifying the shared

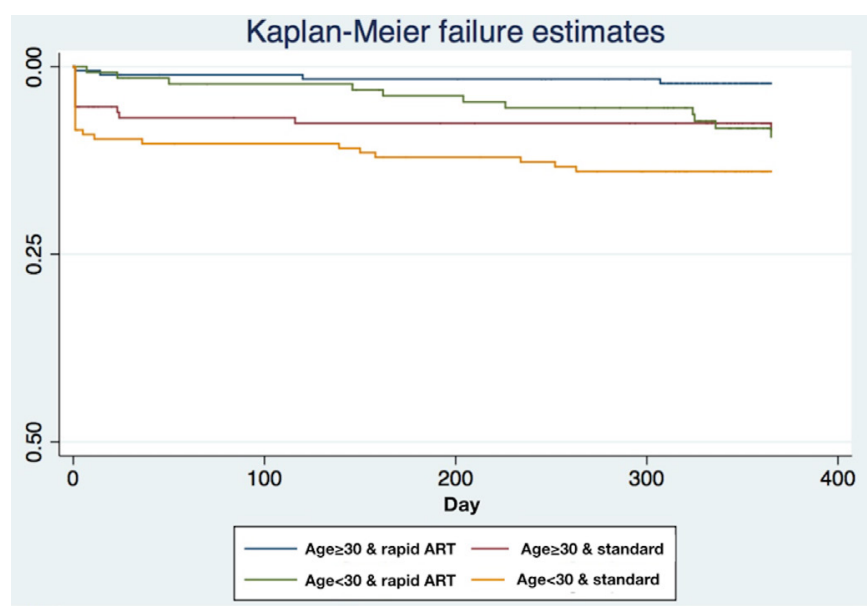

Figure 4 Kaplan-Meier analysis of loss to follow-up stratified by age $<30$ years and timing of ART initiation. ART, antiretroviral therapy. decision-making process between patients and HIV care providers regarding ART initiation. Patients who adopted rapid ART initiation might have been more willing to engage and continue their HIV treatment due to lower CD4 counts, presentation of acute HIV infection or other unreported personal reasons (online supplementary table 1).

Several studies in developed countries have demonstrated faster viral suppression after rapid ART initiation. ${ }^{13} 2021$ In the San Francisco RAPID Study, ${ }^{13}$ the uptake rate of rapid ART was only $43 \%$ in the non-intervention group. The median time to viral suppression was 170 days in the non-intervention group; by contrast, it was 65 days in the RAPID group. In our study, the proportion of patients adopting rapid ART initiation increased from $34 \%$ in 2014 to $68 \%$ in 2017. Apart from the interval between HIV diagnosis and ART initiation, faster viral suppression is also associated with the choice of ART regimen. Our patients without rapid ART initiation were included mainly in the pre-InSTI era, when the multitablet regimens of non-nucleoside reverse transcriptase inhibitor-based or protease inhibitor-based ART remained the mainstay of therapy in Taiwan; these regimens are associated with a higher rate of adverse effects $^{29}$ and poorer adherence ${ }^{3031}$ compared with other regimens. In cohort studies conducted on InSTI-based rapid ART initiation, ${ }^{1320} 2179 \%$ of patients could achieve viral suppression after 3 months of treatment, ${ }^{20}$ and the median time to viral suppression was only 65 days. ${ }^{13}$ In our study, the proportion of patients taking InSTI-based cART increased sharply to $69.8 \%$ in 2017 after a coformulated InSTI-based regimen was introduced in Taiwan in 2016. The time from diagnosis to viral suppression also decreased to 47 days in patients included in 2017, which might potentially decrease the risk of onwards HIV transmission within 2 months after rapid ART initiation.

A high rate (6.6 per 100 PYFU) of incident STIs has been found among patients with acute HIV infection ${ }^{7}$ and patients who continued to practice condomless anal intercourse after being diagnosed as having HIV infection. ${ }^{32}$ In this study, the incidence of any STI before the 
achievement of viral suppression was higher in patients without rapid ART initiation than it was in those with rapid ART initiation (28.3 vs 18.8 per 100 PYFU) within a short follow-up duration; this thus raises concerns that STIs may facilitate HIV transmission through genital secretions with relatively high HIV RNA loads. ${ }^{33} 34$ Notably, although the PARTNER 1 and expanded PARTNER 2 studies have provided robust evidence for treatment as prevention against linked HIV transmission between serodifferent MSM, ${ }^{89}$ the upper bound of the $95 \% \mathrm{CI}$ for HIV transmission among individuals with an STI was 3.17 per 100 couple-years of follow-up; this suggests that faster viral suppression through rapid ART initiation and constant counselling for safe sex among such sexually active patients are urgently required before viral suppression is confirmed. In our study, patients aged $<30$ years were more likely to have incident STIs (aHR: 2.78; $95 \%$ CI 1.53 to 5.06; online supplementary table 5) and LTFU (aHR: 2.19; 95\% CI 1.20 to 3.98; table 4) than did other patients. Notably, we observed that rapid ART initiation (HR: $0.45 ; 95 \%$ CI 0.2 to $0.83 ; \mathrm{p}=0.01$ ) might counterbalance the risk of LTFU among patients aged $<30$ years (figure 4 ) .

Our study has several limitations. First, the intervals between HIV diagnosis or ART initiation and HIV suppression could not be precisely estimated because blood testing was performed at 1 month and subsequently every 3 months according to the national HIV treatment guidelines. In real-world experience, the intervals may vary widely due to many logistic reasons. Second, detection bias for incident STIs might have occurred based on clinical judgments among the care providers. Moreover, this study recorded only symptomatic STIs among patients engaged in HIV care, which would likely underestimate the incidence rates of STIs. Third, our study results might not be generalisable to other healthcare settings that have different experiences with HIV care and adopt different approaches to ART initiation. Fourth, linked HIV transmission could not be documented by this single-centre retrospective study; therefore, the benefit of rapid ART initiation in reducing HIV transmission at the population level could not be directly confirmed.

In conclusion, we determined that rapid ART initiation in the real-world setting was associated with a higher rate of engagement in care, lower LTFU and faster viral suppression. Our findings suggest that scaling up of rapid ART initiation in accordance with the WHO recommendation is feasible in a public sector facility, particularly among younger patients who are more likely to be LTFU and acquire STIs.

\footnotetext{
Author affiliations

${ }^{1}$ Department of Internal Medicine, National Taiwan University Hospital Hsin-Chu Branch, Hsin-Chu, Taiwan

${ }^{2}$ Department of Internal Medicine, National Taiwan University Hospital and National Taiwan University College of Medicine, Taipei, Taiwan

${ }^{3}$ Department of Medicine, National Taiwan University Hospital Jin-Shan Branch, New Taipei City, Taiwan
}

${ }^{4}$ Department of Tropical Medicine and Parasitology, National Taiwan University College of Medicine, Taipei

, Taiwan

${ }^{5}$ Department of Internal Medicine, National Taiwan University Hospital Yun-Lin Branch, Yun-Lin

, Taiwan

${ }^{6}$ Center of Infection Control, National Taiwan University Hospital, Taipei, Taiwan ${ }^{7}$ Department of Medical Research, China Medical University Hospital, Taichung, Taiwan

${ }^{8}$ China Medical University, Taichung, Taiwan

Acknowledgements Patient advisors were not involved in the study design or conduction. Nevertheless, we thank all patients, their families and members of LGBT HIV/AIDS advocacy and support groups in empowering all people living with HIV. We also thank colleagues in the Division of Infectious Diseases, Department of Internal Medicine, National Taiwan University Hospital, for their support and care for people living with HIV at the hospital.

Contributors $\mathrm{Y}-\mathrm{CH}, \mathrm{H}-\mathrm{YS}$ and $\mathrm{C}-\mathrm{CH}$ performed the research. $\mathrm{H}-\mathrm{YS}, \mathrm{Y}-\mathrm{CC}, \mathrm{Y}-\mathrm{SH}$, $\mathrm{K}-\mathrm{YL}, \mathrm{S}-\mathrm{HH}, \mathrm{G}-\mathrm{JC}$ and C-CH were devoted to patient care. Y-CH, Y-ZL, P-YW and $\mathrm{W}-\mathrm{CL}$ collected and analysed the data. $\mathrm{Y}-\mathrm{CH}$ and $\mathrm{C}-\mathrm{CH}$ wrote the paper. $\mathrm{C}-\mathrm{CH}$ and S-CC oversaw the study.

Funding The authors have not declared a specific grant for this research from any funding agency in the public, commercial or not-for-profit sectors.

Competing interests $\mathrm{C}-\mathrm{CH}$ received research support from Gilead Sciences, Merck and ViiV Healthcare and speaker honoraria from Gilead Sciences and ViiV Healthcare and served on the advisory boards for Gilead Sciences and ViiV Healthcare.

Patient consent for publication Not required.

Ethics approval The study was approved by the Research Ethics Committee of National Taiwan University Hospital (Registration No. 201003112R).

Provenance and peer review Not commissioned; externally peer reviewed.

Data availability statement Data are available upon reasonable request.

Open access This is an open access article distributed in accordance with the Creative Commons Attribution Non Commercial (CC BY-NC 4.0) license, which permits others to distribute, remix, adapt, build upon this work non-commercially, and license their derivative works on different terms, provided the original work is properly cited, appropriate credit is given, any changes made indicated, and the use is non-commercial. See: http://creativecommons.org/licenses/by-nc/4.0/.

\section{REFERENCES}

1. WHO Guidelines Approved by the Guidelines Review Committee. Guideline on when to start antiretroviral therapy and on pre-exposure prophylaxis for HIV. 2015. Geneva: World Health Organization Copyright (c) World Health Organization, 2015.

2. Danel C, Moh R, Gabillard D, et al. A trial of early antiretrovirals and isoniazid preventive therapy in Africa. N Engl J Med 2015;373:808-22.

3. Lundgren JD, Babiker AG, Gordin F, et al. Initiation of antiretroviral therapy in early asymptomatic HIV infection. N Engl J Med 2015;373:795-807.

4. Borges Álvaro H, Neuhaus J, Babiker AG, et al. Immediate antiretroviral therapy reduces risk of infection-related cancer during early HIV infection. Clin Infect Dis 2016;63:1668-76.

5. O'Connor J, Vjecha MJ, Phillips AN, et al. Effect of immediate initiation of antiretroviral therapy on risk of severe bacterial infections in HIV-positive people with CD4 cell counts of more than 500 cells per $\mu \mathrm{L}$ : secondary outcome results from a randomised controlled trial. The Lancet HIV 2017;4:e105-12.

6. Cohen MS, Chen YQ, McCauley M, et al. Antiretroviral therapy for the prevention of HIV-1 transmission. New England Journal of Medicine 2016;375:830-9.

7. Cope AB, Crooks AM, Chin T, et al. Incident sexually transmitted infection as a biomarker for high-risk sexual behavior after diagnosis of acute HIV. Sex Transm Dis 2014;41:447-52.

8. Rodger AJ, Cambiano V, Bruun T, et al. Sexual activity without condoms and risk of HIV transmission in Serodifferent couples when the HIV-positive partner is using suppressive antiretroviral therapy. JAMA 2016;316. 
9. Rodger AJ, Cambiano V, Bruun T, et al. Risk of HIV transmission through condomless sex in serodifferent gay couples with the HIVpositive partner taking suppressive antiretroviral therapy (partner): final results of a multicentre, prospective, observational study. The Lancet 2019;393:2428-38.

10. WHO. Hiv/Aids data and statistics, 2018. Available: https://www.who. int/hiv/data/2017_hiv-incidence-2000-2030.png?ua=1 [Accessed 12 Dec 2018].

11. Minn AC, Kyaw NTT, Aung TK, et al. Attrition among HIV positive children enrolled under integrated HIV care programme in Myanmar: 12 years cohort analysis. Glob Health Action 2018;11:1510593.

12. Rosen S, Fox MP. Retention in HIV care between testing and treatment in sub-Saharan Africa: a systematic review. PLoS Med 2011;8:e1001056.

13. Pilcher CD, Ospina-Norvell C, Dasgupta A et al. The effect of sameday observed initiation of antiretroviral therapy on HIV viral load and treatment outcomes in a US public health setting. J Acquir Immune Defic Syndr 2017;74:44-51.

14. Amanyire G, Semitala FC, Namusobya J, et al. Effects of a multicomponent intervention to streamline initiation of antiretroviral therapy in Africa: a stepped-wedge cluster-randomised trial. The Lancet HIV 2016;3:e539-48.

15. Rosen S, Maskew M, Fox MP, et al. Initiating Antiretroviral Therapy for HIV at a Patient's First Clinic Visit: The RapIT Randomized Controlled Trial. PLoS Med 2016;13:e1002015.

16. Labhardt ND, Ringera I, Lejone TI, et al. Effect of offering same-day art vs usual health facility referral during home-based HIV testing on linkage to care and viral suppression among adults with HIV in Lesotho. JAMA 2018:319:1103-12.

17. Langwenya N, Phillips TK, Brittain K, et al. Same-Day antiretroviral therapy (art) initiation in pregnancy is not associated with viral suppression or engagement in care: a cohort study. J Int AIDS Soc 2018;21:e25133.

18. Koenig SP, Dorvil N, Dévieux JG, et al. Same-Day HIV testing with initiation of antiretroviral therapy versus standard care for persons living with HIV: a randomized unblinded trial. PLoS Med 2017; 14:e1002357.

19. WHO Guidelines Approved by the Guidelines Review Committee. Guidelines for managing advanced HIV disease and rapid initiation of antiretroviral therapy. Geneva: World Health Organization, 2017.

20. Hoenigl M, Chaillon A, Moore DJ, et al. Rapid HIV viral load suppression in those initiating antiretroviral therapy at first visit after HIV diagnosis. Sci Rep 2016;6.

21. Girometti N, Nwokolo N, McOwan A, et al. Outcomes of acutely HIV1-infected individuals following rapid antiretroviral therapy initiation. Antivir Ther 2016;22:77-80.
22. CDC T. Hiv/Aids prevention and control manual. 3rd edn. Taiwan Centers for Disease Control, 2014: 37-41.

23. CDC T. Diagnosis of HIV infection 2018:1-4.

24. Chi BH, Yiannoutsos CT, Westfall AO, et al. Universal definition of loss to follow-up in HIV treatment programs: a statistical analysis of 111 facilities in Africa, Asia, and Latin America. PLoS Med 2011;8:e1001111.

25. Tweya H, Feldacker C, Estill J, et al. Are They Really Lost? "True" Status and Reasons for Treatment Discontinuation among HIV Infected Patients on Antiretroviral Therapy Considered Lost to Follow Up in Urban Malawi. PLoS One 2013;8:e75761.

26. Ford N, Migone C, Calmy A, et al. Benefits and risks of rapid initiation of antiretroviral therapy. AIDS 2018;32:17-23.

27. Maughan-Brown B, Smith P, Kuo C, et al. Readiness for antiretroviral therapy: implications for linking HIV-infected individuals to care and treatment. AIDS Behav 2018;22:691-700.

28. Fehr J, Nicca D, Goffard J-C, et al. Reasons for not starting antiretroviral therapy in HIV-1-infected individuals: a changing landscape. Infection 2016;44:521-9.

29. PY W, Cheng CY, Liu CE, et al. Multicenter study of skin rashes and hepatotoxicity in antiretroviral-naive HIV-positive patients receiving non-nucleoside reverse-transcriptase inhibitor plus nucleoside reverse-transcriptase inhibitors in Taiwan. PLoS One 2017;12:e0171596.

30. Sutton SS, Hardin JW, Bramley TJ, et al. Single- versus multipletablet HIV regimens: adherence and hospitalization risks. The American journal of managed care 2016;22:242-8.

31. Jacobson K, Ogbuagu O. Integrase inhibitor-based regimens result in more rapid virologic suppression rates among treatmentnaïve human immunodeficiency virus-infected patients compared to non-nucleoside and protease inhibitor-based regimens in a real-world clinical setting: a retrospective cohort study. Medicine 2018;97:e13016.

32. Gorbach PM, Javanbakht M, Bolan RK. Behavior change following HIV diagnosis: findings from a cohort of Los Angeles MSM. AIDS Care 2018;30:300-4.

33. Dyer JR, Eron JJ, Hoffman IF, et al. Association of CD4 cell depletion and elevated blood and seminal plasma human immunodeficiency virus type 1 (HIV-1) RNA concentrations with genital ulcer disease in HIV-1-Infected men in Malawi. $J$ Infect Dis 1998;177:224-6.

34. Sadiq STet al. The effects of urethritis on seminal plasma HIV-1 RNA loads in homosexual men not receiving antiretroviral therapy. Sex Transm Infect 2005;81:120-3. 